\title{
The Relationship Between Retinal Nerve Fiber Layer Thickness and Optic Nerve Head Neuroretinal Rim Tissue in Glaucoma
}

\author{
Nimesh B. Patel, ${ }^{1}$ Michael Sullivan-Mee, ${ }^{2}$ and Ronald S. Harwerth ${ }^{1}$ \\ ${ }^{1}$ Department of Vision Sciences, University of Houston College of Optometry, Houston, Texas, United States \\ ${ }^{2} \mathrm{New}$ Mexico Veterans Affairs Health Care System, Albuquerque VA Medical Center, Albuquerque, New Mexico, United States
}

Correspondence: Nimesh B. Patel, Department of Vision Sciences, University of Houston College of Optometry, $505 \mathrm{~J}$. Davis Armistead Building, Houston, TX 77204, USA; npatel@optometry.uh.edu.

Submitted: February 18, 2014 Accepted: September 13, 2014

Citation: Patel NB, Sullivan-Mee M, Harwerth RS. The relationship between retinal nerve fiber layer thickness and optic nerve head neuroretinal rim tissue in glaucoma. Invest Ophthalmol Vis Sci. 2014;55:6802-6816. DOI:10.1167/ iovs.14-14191
Purpose. The purpose of this study was to determine the relationship between optical coherence tomography (OCT) measures of retinal nerve fiber layer (RNFL) and neuroretinal rim (NRR) in a nonhuman primate experimental glaucoma model, and in a population of clinical patients.

МетноDs. For nonhuman primates, normative data were collected from 44 healthy monkeys, and nine animals with unilateral experimental glaucoma that were followed longitudinally. Cross-sectional human subjects data were collected from 89 healthy, 74 glaucoma suspects, and 104 glaucoma patients. Individualized transverse scaling for OCT scans was calculated using a schematic eye that incorporated optical ocular biometry. Custom algorithms were used to quantify RNFL thickness with and without vessels removed, scaled minimum rim width (sMRW), and neural rim volume (NRV).

Results. For the experimental glaucoma group, NRR parameters showed the first changes with increased cumulative IOP. The data for both NRR and RNFL measures were best fit by an exponential rise model (NRV, $R^{2}=0.79, P<0.01$, sMRW, $R^{2}=0.74, P<0.01$ ). The major retinal vascular thickness contribution to the RNFL decreased $(0.03 \mu \mathrm{m} / \mu \mathrm{m}, P<0.01)$ with RNFL loss, but the percent vascular contribution increased $(-0.1 \% / \mu \mathrm{m}, P<0.01)$ with disease progression. Overall, the findings for the cross-sectional human data were similar to those of the experimental model.

ConcLusions. The findings illustrate a nonlinear relationship between NRR and RNFL measures and provide support for the use of multiple OCT scaled morphological measures for the diagnosis and management of primary open angle glaucoma in humans.

Keywords: glaucoma, RNFL, neuroretinal rim, optical coherence tomography
The glaucomas are progressive optic neuropathies that can result in irreversible blindness. ${ }^{1}$ As the world's population ages and lives longer, the incidence and prevalence of glaucoma are expected to increase. Because early detection and adequate IOP reduction can slow progression and reduce the burden of disease, ${ }^{2-4}$ there should be an emphasis on early disease detection. At the present time, clinical diagnosis and management of glaucoma are based on an assessment of IOP, perimetric visual field testing, and ophthalmoscopic evaluation of the optic nerve head $(\mathrm{ONH})$ and nerve fiber layer $(\mathrm{NFL}) .^{5-7}$

The functional loss of visual sensitivity in glaucoma is a direct result of structural damage to retinal ganglion cells (RGCs). ${ }^{8,9}$ Although standard automated perimetry (SAP) measures of visual sensitivity provide valuable information about the stage of glaucoma, inter- and intrasubject variability make it difficult to reliably detect small changes, especially in early glaucoma, often requiring a confirmatory test. ${ }^{10}$ Similarly, there is large variability in the ophthalmoscopic evaluation of the ONH and NFL, due to both broad interindividual anatomic variation and the subjective nature of the test. ${ }^{11,12}$ However, the relatively recent development and advancement of in vivo imaging, especially with optical coherence tomography (OCT), has substantially improved the quantification, reliability, and repeatability, of posterior segment structures for diagnosis and management of glaucoma. ${ }^{13-18}$ With this technology, the morphology of the ONH and thickness of the circumpapillary retinal nerve fiber layer (RNFL) can be quantified using OCT scans centered on the optic nerve head.

Clinically, the circumpapillary RNFL thickness, sampled at a 6-degree radius from the center of the $\mathrm{ONH}$, has become the mainstay for glaucoma assessment because it includes most of the RGC axons in the retina. However, these axons also make up the neuroretinal rim (NRR) tissue of the $\mathrm{ONH}$, and recently, measures of the NRR tissue, including the minimum rim width (MRW), horizontal rim width, rim area (RA), and neural rim volume (NRV), have been shown to be important measures for glaucoma diagnosis. ${ }^{19-21}$ Although both the RNFL and NRR measures, in addition to RGC axons, include significant nonneuronal components, including glial and vascular tissue, ${ }^{22-24}$ there should be a strong correlation between these measures in normal healthy eyes and across all stages of glaucomatous neuropathy.

The laser-induced elevation of IOP as an experimental model of glaucoma in the macaque monkey has been important for investigations of both structural and functional changes ${ }^{25-27}$ that demonstrates increased ONH cupping, ${ }^{28-30}$ loss of RNFL 
thickness, and visual sensitivity, ${ }^{31}$ similar to those observed in human glaucoma. In a recent study investigating deep and surface $\mathrm{ONH}$ morphology and RNFL anatomy, ONH changes were found to precede RNFL and multifocal electroretinogram changes in early experimental glaucoma. ${ }^{32}$ However, the relationship between these RGC axon-containing structures were not fully described in this longitudinal data set. In addition, the relationships between RNFL and NRR parameters in humans have been reported only in cross-sections of glaucoma patients, ${ }^{33}$ where the relationship was shown to be linear in nature.

Given that methods of quantifying NRR parameters using OCT imaging will have considerable clinical application, it is important to understand how the NRR changes over the time course of glaucoma and how NRR and RNFL measurements correlate across stages of neuropathy. Therefore, the primary purpose of this study was to determine the relationship between NRR morphology and circumpapillary RNFL thickness over the time course of experimental glaucoma in nonhuman primates, and in cross-sectional data from healthy, glaucoma suspect, and glaucoma human patients. To make these comparisons, the methodology for both species involved custom algorithms for correcting for transverse magnification based on the optical properties of each eye. Portions of this work were presented in a dissertation, and as an ARVO abstract (Patel NB, et al. IOVS 2013;54:ARVO EAbstract 3154).

\section{Methods}

\section{Animal Subjects}

Normative cross-sectional data were collected from 44 healthy rhesus monkeys (Macaca mulatta), and longitudinal data were collected from nine animals with unilateral, laser-induced experimental glaucoma. All animals maintained good systemic health throughout the experimental period. Data from only one eye of each animal was used for the normative database, whereas both eyes of the animals with experimental glaucoma were analyzed, to assess for repeatability (control eye) and to detect disease progression. Experimental and animal care procedures were reviewed and approved by the Institutional Animal Care and Use Committee of the University of Houston. The use of animals for these experiments adhered to the ARVO Statement for the Use of Animals in Ophthalmic and Vision Research and to the National Institutes of Health guidelines for the care and use of laboratory animals.

\section{Animal Preparation}

Before conducting laser or scanning procedures, the monkeys were anesthetized with an intramuscular injection of ketamine $(20-30 \mathrm{mg} / \mathrm{kg})$ and xylazine $(0.8-0.9 \mathrm{mg} / \mathrm{kg})$ and treated with a subcutaneous injection of atropine sulfate $(0.04 \mathrm{mg} / \mathrm{kg})$. Throughout the experiment, body temperature was monitored and maintained using a thermal blanket (TC 1000 temperature controller; CWE, Ardmore, PA, USA), while heart rate and pulse were monitored with a pulse oximeter (model $9847 \mathrm{~V}$; Nonin Medical, Inc., Plymouth, MN, USA). For spectral-domain (SD) OCT imaging, the animal's pupils were dilated with $1 \%$ tropicamide, an eyelid speculum was used to keep the eye open, and a plano power custom-designed rigid gas-permeable contact lens was placed on the eye to prevent corneal dehydration, and maintain optical clarity.

In the nonhuman primate, an acute elevation of IOP has been shown to result in a decrease in optic nerve rim measures. ${ }^{34}$ Hence, to minimize these compression effects, 1 drop of $1 \%$ apraclonidine or $0.2 \% / 0.5 \%$ brimonadine tartrate/ timolol maleate was instilled in the experimental glaucoma eye, when IOP measures were greater than $25 \mathrm{~mm} \mathrm{Hg}$. The threshold of $25 \mathrm{~mm} \mathrm{Hg}$ was used based on anesthesia pressure-lowering effects of xylazine, ${ }^{35,36}$ with the aim of scanning the experimental glaucoma optic nerve when the IOP was $20 \mathrm{~mm} \mathrm{Hg}$ or less. During the imaging and laser procedures, the monkey's head was stabilized using mouth and occipital bars.

\section{Laser-Induced Ocular Hypertension}

Experimental glaucoma was induced by scaring the trabecular meshwork using argon laser energy delivered through a standard ophthalmic slit lamp system (HGM PC endo; HGM, Salt Lake City, UT, USA). Contiguous argon laser (1.0 W, 0.5 seconds, $50 \mu \mathrm{m}$ ) burns were applied to the trabecular meshwork through a laser gonioscopy lens designed for a nonhuman primate eye (Ocular Kaufman; Ocular Instruments, Bellevue, WA, USA). ${ }^{37-40}$ The initial procedure involved 270 degrees of the drainage angle, followed by retreatment of 180 degrees at 4-week intervals until sustained elevated IOPs were achieved. Intraocular pressure measurements were made using a Tono-Pen XL (Reichert, Inc., Depew, NY, USA) at each scanning session, and within 10 minutes of administering anesthesia. ${ }^{35}$ The IOP difference between the two eyes was interpolated over time and used to calculate a cumulative IOP, expressed in $\mathrm{mm} \mathrm{Hg.days,} \mathrm{referenced} \mathrm{to} \mathrm{the} \mathrm{first} \mathrm{laser}$ treatment. $^{38,41}$

\section{Human Subjects}

Scans from one randomly selected eye of 265 human subjects were analyzed for this study. A total of 180 of the subjects were recruited at the Albuquerque VA Medical Center and were subjects for an ongoing prospective, longitudinal observational glaucoma research study; 85 subjects were recruited from the University of Houston, University Eye Institute. The study adhered to the tenets of the declaration of Helsinki, and all aspects of the study were reviewed by either the University of New Mexico Institutional Review Board or the University of Houston Committee for Protection of Human Subjects.

Enrollment criteria included open angles as determined by slit lamp examination for healthy subjects, and documented with gonioscopy for glaucoma and glaucoma suspect patients. Subjects could not have had any active or prior corneal or iris pathology, and only individuals with no previous history of ocular surgery, other than uncomplicated cataract extraction, were recruited. Individuals with a history of angle closure, secondary glaucoma, optic neuropathy, or nonglaucomatous visual pathway anomalies were excluded. To minimize any axial length effects on the $\mathrm{ONH}$, only individuals with ametropia less than 5 diopters were included.

For this study, normal was defined as a subject who, on dilated ophthalmoscopy had a normal-appearing $\mathrm{ONH}$ with no asymmetry between the two eyes, no visual field defects as determined with standard automated perimetry, and IOP lower than $21 \mathrm{~mm} \mathrm{Hg}$. Glaucoma suspects were defined as individuals with no visual field defects and (1) optic nerves that looked suspicious, but without NFL defect as noted with ophthalmoscopy, or (2) ocular hypertensive individuals with a history of IOP higher than $21 \mathrm{~mm} \mathrm{Hg}$ and normal ONH and NFL appearance, with no progression noted to the extent of the clinical history available. Glaucoma subjects were recruited if they had typical glaucomatous $\mathrm{ONH}$ changes with corresponding reproducible field loss and treated IOP in the target range. 

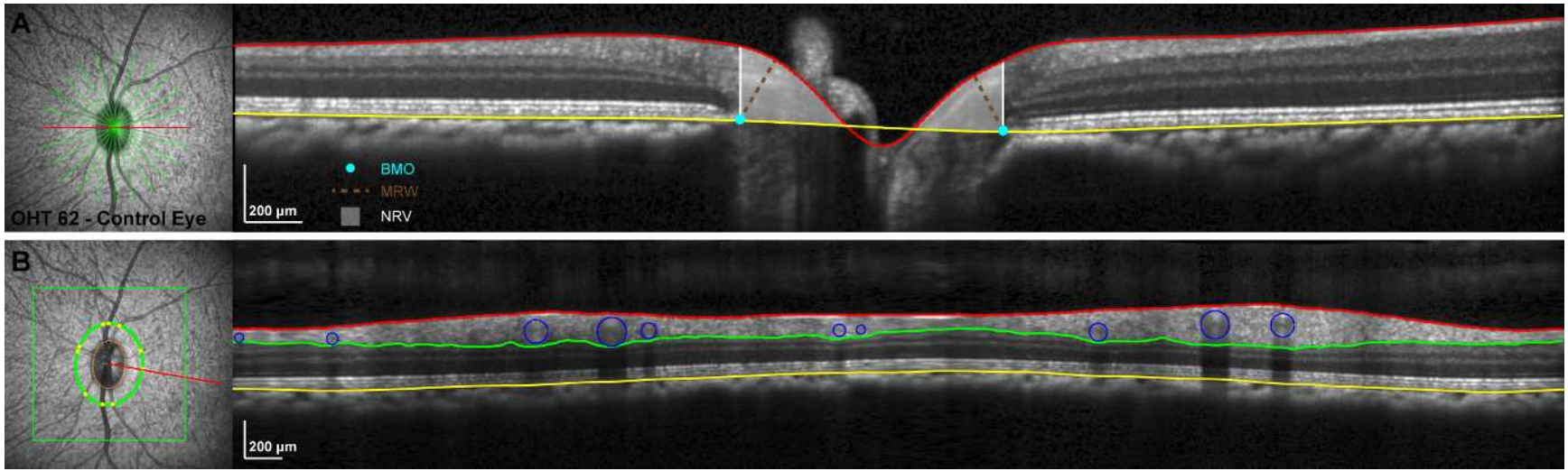

Figure 1. Control eye data from one nonhuman primate illustrating the OCT morphological measures used for data analysis. (A) The OCT B-scan corresponds to the horizontal section in red on the IR-SLO image. The BMO was marked on each radial OCT B-scan, and used as a reference for measuring the NRV and the MRW in scaled images. (B) The BMO points were transposed to the SLO image of the raster scan and fit with an ellipse (orange). This was then used as a reference to interpolated B-scans $550 \mu \mathrm{m}$ from the BMO (green) to measure RNFL thickness. The yellow markings on the elliptical scan path correspond to the major retinal vasculature identified and removed in the OCT B-scan. Retinal nerve fiber layer B-scans were referenced to a plane connecting the center of the BMO and the fovea (red line).

\section{Optical Coherence Tomography}

All SD OCT scans were acquired using the Spectralis HRA+OCT system (Heidelberg Engineering, Heidelberg, Germany), after pharmacological dilation of the pupils. For nonhuman primates, SD-OCT scans used for data analysis included (1) 12-line, 20-degree radial scan, and (2) a 49- or 97-line, $20 \times 20$ degree raster scan centered on the optic nerve. The lasered eye, in the experimental glaucoma group, was always scanned second, allowing for maximum pressure reduction. ${ }^{36}$ For human subjects, scans included (1) 12-line, 20-degree radial scan, and (2) a 12-degree circumpapillary scan centered on the optic nerve. For improved scan quality, all scans were acquired with eye tracking and averaging was set to at least 16 frames. The OCT scan data were exported as raw (.vol) files and analyzed using custom software (MATLAB; The MathWorks, Inc., Natick, MA, USA).

\section{Ocular Biometry and Retinal Scaling}

Ocular biometry, including corneal curvature, and axial length were obtained using optical biometry, with either the Lenstar LS900 (Haag-Streit, Koeniz, Switzerland) or IOL Master (Zeiss Meditec, Dublin, CA, USA). Crystalline lens thickness and curvature were interpolated from published normative data. ${ }^{42-44}$ These parameters were incorporated into a threesurface schematic eye and the transverse scaling for OCT scans was calculated from the second nodal plane to the retina, assuming a spherical retinal surface. ${ }^{45}$ Additional details on the scaling methodology have been previously described. ${ }^{24,46,47}$ No adjustments were made to axial scaling, which is dependent on the specifications of the imaging system.

\section{Optic Nerve Head Analysis}

The outer dimensions of the $\mathrm{ONH}$ rim margin were determined in three dimensions by marking the RPE/Bruch's membrane opening $(\mathrm{BMO})^{48,49}$ on each side of the $\mathrm{ONH}$ on the 12 radial B-scans and fitting the resultant 24 points with an ellipse (Fig. 1A). The border for the inner limiting membrane (ILM) was manually delineated, avoiding any central vascular hyaloid remnants or Bergmeister's papilla that were most prominent and commonly noted in nonhuman primate eyes. Bruch's membrane was marked up to the opening, and a spline fit was used to connect the segmentation between the BMO points, corresponding to the opening of the neural canal. On each B-scan, scaled to a 1:1 aspect ratio, the shortest distance from the end of Bruch's membrane to the ILM within the $\mathrm{ONH}$ region was used to calculate the MRW as described by Reis and colleagues, ${ }^{50,51}$ and the average MRW was used for all analysis.

Similar to the previously reported relationship between rim width and disc diameter using planimetry ${ }^{52}$ and MRW and BMO area ${ }^{19}$ in human subjects, ${ }^{49}$ there was a significant relationship between average MRW and the BMO ellipse circumference (normal human subjects, slope $=-0.05 \mu \mathrm{m} /$ $\mu \mathrm{m}, R^{2}=0.21, P<0.01$, normal nonhuman subjects, slope $=$ $\left.-0.04 \mu \mathrm{m} / \mu \mathrm{m}, R^{2}=0.18, P<0.01\right)$. As this relationship represents the distribution of tissue across varying disc size, rather than axonal content, ${ }^{53}$ a scaled MRW (sMRW) was calculated for each subject, using similar principals as those for scaling RNFL thickness measures (Equation 1). Specifically, the raw average MRW was multiplied with the BMO ellipse circumference and then divided by a rounded average BMO ellipse circumference of $4.8 \mathrm{~mm}$ for human eyes or $4.1 \mathrm{~mm}$ for nonhuman primate eyes. These average BMO circumferences were based on measures collected to date in our laboratory.

$$
\text { sMRW }=\frac{\text { average } M R W \times B M O \text { Ellipse Circumference }}{4.8 \mathrm{~mm} \text { or } 4.1 \mathrm{~mm}}
$$

To calculate the NRV, a total retinal thickness map from the ILM and BM segmentation of the radial scans was first created, from three dimensional representations of both surfaces. This thickness map was then scaled to 1 pixel:1 $1 \mu \mathrm{m}$, and the tissue/ pixel content above the splined BM plane within the threedimensional BMO plane was used to compute the NRV.

\section{Retinal Nerve Fiber Layer Analysis: Nonhuman Primate}

Nonhuman primate eyes have a significantly shorter axial length $(19 \mathrm{~mm})$ than human eyes $(24 \mathrm{~mm})$, and interindividual differences in ocular biometry can have larger effects on the scan path and dimension. ${ }^{42,46}$ In addition, the nonhuman primate $\mathrm{ONH}$ is smaller and more elliptical compared with that of a human. Hence, an elliptical scan path $550 \mu \mathrm{m}$ from the BMO was used for RNFL quantification (Fig. 1B). This distance 
from the BMO was selected based on previous work that demonstrated the best relationship with the standard 12degree circular scan in nonhuman primates. ${ }^{46}$

For accurate anatomical correspondence, the BMO marked for radial scans was transposed to the raster scan after registering the two scanning laser ophthalmoscope (SLO) images using a generalized dual bootstrap iterative closestpoint algorithm (i2k retina; DualAlign LLC, Clifton, NY, USA). The transposed BMO was used to construct an ellipse $550 \mu \mathrm{m}$ from its margin that was then used to interpolate a B-scan from the raster scan data. The resultant B-scan was then scaled to a 1:1 aspect ratio and the ILM and RNFL/RGC layer borders were identified using a custom algorithm. ${ }^{46}$ Segmentation errors, which were most common around major retinal vasculature that cast shadows on the underlying tissue, were then manually corrected. When visible, any vessel contributing to the RNFL thickness was included in its entirety for thickness measures. Using the raster scan SLO image and B-scan shadows as a guide, the borders of each major vessel were marked, and the circular region corresponding to each vessel was subsequently subtracted from the RNFL thickness. Specifics for the scan interpolation and segmentation methodology have been presented elsewhere. ${ }^{46}$

\section{Retinal Nerve Fiber Layer Analysis: Human Subjects}

The B-scan from the 12-degree circular scan centered on the $\mathrm{ONH}$ and the instrument-determined segmentation of the RNFL was imported into MATLAB. Scans were scaled to a 1:1 aspect ratio using the individualized calculated transverse scaling. Errors in segmentation were manually corrected, and similar to nonhuman primate analysis, all vessels contributing to the RNFL thickness were included. The major retinal vessels were then identified with the aid of the infrared (IR)-SLO image with an overlay of the scan path, and shadows cast on the underlying retina in the B-scans. Subsequently, circular regions corresponding to the retinal vasculature were subtracted from the RNFL thickness.

The OCT scan path is dependent on the optics of the eye, and scans farther from the rim margin result in thinner RNFL measures. Although there is a significant relationship between axial length and RNFL thickness, this does not accurately represent a change in axon content, but is a reflection of the dispersion of the nerve fiber layer. ${ }^{54-57}$ With the assumption that the nerve fiber layer cross-sectional area (RNFL Thickness $\times$ Scan Circumference) remains constant in the circumpapillary region, RNFL thickness measures can be rescaled when retinal transverse scaling is known. ${ }^{24,55,58,59}$ For global measures, the scaled RNFL thickness with and without major retinal vascular contribution was calculated using Equation 2, and a nominal scan circumference for an emmetropic eye of $10.9 \mathrm{~mm}$.

\section{Scaled RNFL thickness}

$=\frac{\text { RNFL Thickness } \times \text { Calculated Scan Circumference }}{10.9 m m}$

All statistical analysis and plots reported were using either GraphPad Prism version 6.0 (GraphPad Software, La Jolla, CA, USA), MATLAB and Statistics Toolbox (The MathWorks, Inc.), or SigmaPlot 12.0 (Systat Software, San Jose, CA, USA). Nonlinear fits were used only when a linear fit did not describe the data well, as determined by a runs test, and a nonlinear fit was supported by Akaike information criteria (AICc) analysis. ${ }^{60}$

\section{Results}

\section{Nonhuman Primate Subjects}

Normative data were collected from 44 monkeys with an age range from 1 month to 23 years of age (median $=5.47$ years, interquartile range 1.07-8.64 years), and average axial length of $18.26 \pm 1.9 \mathrm{~mm}$. An additional nine monkeys with experimental glaucoma (age range of 3.00-7.33 years and median age of 5.21 years at the first laser treatment) were followed over the time course from normal to moderate or endstage glaucoma. The longest follow-up was 861 days, whereas the shortest was 286 days after the first laser session. All animals maintained good systemic health and had no other ocular interventions, other than unilateral argon laser scarring of the trabecular meshwork in the experimental group, for the period of this study. Additional details on each experimental glaucoma animal are presented in Table 1.

Data for the range of measures, means, and repeatability for ONH neuroretinal rim morphology and RNFL thicknesses for normal monkey eyes are illustrated in Table 2 . The repeatability $(2.77 \times \mathrm{Sw})$ of each measure was assessed using the estimated within-subject standard deviation (Sw) from the repeated measures from the control eyes of the experimental glaucoma subjects. ${ }^{61}$ Although there is significant intersubject variability in the normative data, there was good repeatability for all measures.

An analysis of the normative data $(n=44)$ revealed an inverse relationship between the BMO area and the raw MRW (slope $=-60 \mu \mathrm{m} / \mathrm{mm}^{2}, R^{2}=0.16, P<0.01$ ). However, when scaled using Equation 1, the sMRW increased with BMO area (slope $=23 \mu \mathrm{m} / \mathrm{mm}^{2}, R^{2}=0.14, P<0.01$ ), as did the NRV (slope $=0.08 \mathrm{~mm}^{3} / \mathrm{mm}^{2}, R^{2}=0.1, P=0.04$ ) and RNFL thickness (slope $=12 \mu \mathrm{m} / \mathrm{mm}^{2}, R^{2}=0.12, P=0.02$ ). None of the morphological measures from B-scans scaled 1:1 aspect ratio, including $\mathrm{BMO}$ area, NRV, sMRW, and RNFL thickness measures with and without major retinal vasculature, was related to axial length $(P>0.1)$.

\section{Human Subjects}

Spectral-domain OCT data were collected from one eye of 265 human subjects. Of these, 89 subjects were healthy, 74 were glaucoma suspects, and 102 had a diagnosis of open angle glaucoma. The mean ages were $63.5 \pm 9.8$ years, $64.4 \pm 9.6$ years, and $68.6 \pm 9.4$ years, respectively. Generally, the open angle glaucoma study patients had early to moderate glaucoma as diagnosed by SAP with the median mean deviation of -3.7 $\mathrm{dB}$, and an interquartile range from $-1.25 \mathrm{~dB}$ to $-7.8 \mathrm{~dB}$, as illustrated in Table 3.

Data for the means and medians for ONH NRR measures and RNFL thickness for normal healthy human eyes, presented in Table 4, are quite similar to the previously reported data for normal subjects, using in vivo imaging. ${ }^{19,62}$ With fixed transverse scaling, there was a significant relationship between axial length and global RNFL thickness, either with major retinal vasculature included in the measurement (slope $\left.=-3.2 \mu \mathrm{m} / \mathrm{mm}, R^{2}=0.14, P<0.01\right)$ or with the retinal vasculature removed (slope $=-2.9 \mu \mathrm{m} / \mathrm{mm}, R^{2}=0.13, P<$ 0.01). When RNFL thickness was scaled using Equation 2, neither global RNFL thickness with (slope $=0.2 \mu \mathrm{m} / \mathrm{mm}, P=$ 0.77), or without (slope $=0.3 \mu \mathrm{m} / \mathrm{mm}, P=0.70$ ) retinal vasculature removed was significantly related to axial length. Although the relationship between the BMO area and raw MRW thickness for healthy subjects was statistically significant ( $n=89$, slope $\left.=-62 \mu \mathrm{m} / \mathrm{mm}^{2}, R^{2}=0.22, P<0.01\right)$, when the MRW was scaled (sMRW), the relationship was not significant (slope $=23 \mu \mathrm{m} / \mathrm{mm}^{2}, R^{2}=0.04, P=0.06$ ) nor was the 
relationship between BMO area and NRV $\left(R^{2}=0.01, P=\right.$ 0.32 ). Similar to the data for nonhuman primates, none of the morphological measures from B-scans scaled 1:1 $\mu \mathrm{m}$, for normal human eyes, including BMO area, NRV, and sMRW, were related to axial length $(P>0.1)$.

\section{Intraocular Pressure and Morphological Measures in the Nonhuman Primate}

The primate model of experimental glaucoma is a high IOP model, and it is reasonable that the signs of optic neuropathy will be related to the time and/or magnitude of the induced pressure. Hence, the effect of IOP insult was evaluated by cumulative IOP, ${ }^{38,41}$ with the relationships between the cumulative IOP of the laser-treated eye and morphological measures assessed with an exponential decay model $(y=a+b$ $\times \mathrm{e}^{(-\mathrm{tx})}$, Fig. 2). Fits to the aggregate grouped data illustrate the similarity in time constant for NRV $(t=0.0013 \pm 0.0001)$ and sMRW ( $t=0.001 \pm 0.0001)$, but a 5-fold smaller constant for RNFL thickness $(t=0.0002 \pm 0.00006)$. These differences in time constants would imply that thinning of the RNFL progresses more slowly than the reduction of ONH NRR tissue in the experimental glaucoma model.

To compare across morphological measures, the data were converted to SD units ( $z$-scores), based on either the intersubject variability from normative data, or the control eye intrasubject variability from repeated measures. For the experimental glaucoma eyes, the onset of a detectable disease state for each animal was determined as the first pressure time point at which a particular measure exceeded two times the intersubject SD from the mean of the normative data on two consecutive scan sessions ( $95 \%$ confidence limit of normative data, Fig. 3A). In comparison, progression, of pressure-induced neuropathy was determined as the cumulative pressure point at which a measure exceeded the repeatability or measurement error on two consecutive scan sessions $(2.77 \times$ Sw; Fig. 3B). Based on the $z$-scores using intrasubject variability, progression could be assessed over a large range for NRV, sMRW, and RNFL (maximum $z$-score changes of $-23.6,-27.2$, and -27.7). Although similar in magnitude, the relationship among these three measures was not linear, as illustrated by the differences in the best fits to the aggregate data and by the relationship of the morphological measures. In the rapidly progressive model of experimental glaucoma, the differences in IOP critical points are small, but for all nine animals, initial progression was first noted with either SMRW $(n=3)$ or both sMRW and NRV ( $n$ $=6$ ) (Fig. 3B), whereas the onset of disease versus no disease was first detected by both sMRW and/or NRV in eight animals, and by RNFL thickness in only one animal (Table 1). These data illustrate differences in axon-containing morphological measures, with disease progression, and are similar to those previously reported for this model..$^{30,32,63}$

\section{Relationship Between ONH NRR Measures}

Both MRW and NRV are measures of ONH NRR tissue, one being an average thickness and the other a total volume, and should be in good agreement. However, whereas NRV includes disc size in its calculation, the raw MRW does not. In addition, because of differences in units of measurement (i.e., linear versus cubed), the relationship between these two measures cannot be linear. To determine the best empirical relationship between the two metrics, the data for normal and glaucoma eyes of monkeys (Figs. 4A, 4C) and human subjects and patients with glaucoma (Figs. 4B, 4D) were analyzed using both raw and scaled MRW. For both datasets, a power function was used to describe the data, and the coefficient of variation was larger for SMRW (nonhuman primate, $R^{2}=0.98$, human 
TABLE 2. Range, Mean, Coefficient of Variation (CV), and Repeatability of OCT-Derived Morphological Measures for Normal Nonhuman Primate Eyes

\begin{tabular}{|c|c|c|c|c|}
\hline & \multicolumn{3}{|c|}{ Normative Data, $n=44$} & \multirow{2}{*}{$\begin{array}{c}\text { Experimental Glaucoma Control Eyes, } n=9 \\
\text { Repeatability, } 2.77 \times \mathbf{S w}\end{array}$} \\
\hline & Range & Mean (SD) & $\mathbf{C V}, \%$ & \\
\hline Rim volume, $\mathrm{mm}^{3}$ & $0.18-0.47$ & $0.29(0.06)$ & 20.1 & 0.031 \\
\hline Scaled MRW, $\mu \mathrm{m}$ & $216.0-404.5$ & $288.5(34.23)$ & 11.9 & 20.57 \\
\hline RNFL thickness, $\mu \mathrm{m}$ & 96.8-138.1 & $124.1(8.89)$ & 7.2 & 5.54 \\
\hline RNFL thickness MRV removed, $\mu \mathrm{m}$ & $87.0-125.4$ & $112.8(8.45)$ & 7.5 & 5.49 \\
\hline Vascular contribution, $\mu \mathrm{m}$ & $8.04-14.79$ & $11.35(1.53)$ & 13.5 & 3.08 \\
\hline
\end{tabular}

subjects, $R^{2}=0.95$ ) than with raw MRW measures (nonhuman primate, $R^{2}=0.95$, human subjects, $R^{2} 0.85$ ).

\section{Vascular Contributions to the RNFL Thickness}

The segmentation methodology used provided a means to assess overall vascular thickness and percent vascular contribution to RNFL thickness at different stages of the disease. The results (Fig. 5) showed that the vascular contribution to the RNFL in normal healthy eyes was $9.16 \% \pm 1.2 \%$ for monkey and $14.0 \% \pm 1.6 \%$ for human subjects. Over the time course of experimental glaucoma, there was a slight but significant decrease in vascular thickness contribution with progression (right to left on the $x$-axis, Fig. 5A). The trend was more pronounced, as illustrated by the steeper slope and larger coefficient of variation for the cross sectional data for human subjects (Fig. 5B). However, when expressed as a percentage of total RNFL thickness, the vascular contribution increased with a decrease in global RNFL thickness, for both nonhuman (Fig. 5C) and human subjects (Fig. 5D) with similar rates of change $(P=0.83)$. Thus, the branches of the central retinal artery and vein make a substantial contribution to global RNFL thickness, which varies with the stage of neuropathy. Hence, all RNFL thickness analyses performed for this study were done after subtracting the thickness attributed to blood vessels to minimize the nonneuronal effects on the results.

\section{Relationship Between ONH NRR and RNFL Measures}

Based on the logic that underlaid the comparison of SMRW and NRV (Fig. 3) (i.e., axons belonging to the same RGCs are found both in the RNFL and the ONH NRR tissue), there also should be a strong relationship between these structures. With progressive loss of neural tissue in the experimental glaucoma model, a corresponding loss in both RNFL and NRR parameters was expected. However, similar to the observations with cumulative IOP, when RNFL measures are plotted as functions of NRR parameters, a change in the ONH NRR tissue is noted before thinning of RNFL (Figs. 6A, 6C). Based on reports that there are neural and nonneuronal remodeling in these two regions, ${ }^{64-67}$ an exponential rise to maximum model $(y=\mathrm{a}+$ $\left.\mathrm{b}\left(1-\mathrm{e}^{-\mathrm{cx}}\right)\right)$ was used to describe the relationship between RNFL thickness and the sMRW thickness $\left(R^{2}=0.74, P<0.01\right)$

TABLE 3. Mean Deviations From 24-2 Sita Standard Visual Fields for Healthy, Glaucoma Suspects, and Glaucoma Patients

\begin{tabular}{llcr}
\hline & Range, $\mathbf{d B}$ & Median, dB & $\begin{array}{c}\text { Interquartile } \\
\text { Range, } \mathbf{d B}\end{array}$ \\
\hline Healthy & +2.59 to -4.69 & -0.27 & 0.42 to -1.36 \\
Glaucoma suspect & +2.36 to -4.03 & -0.34 & 0.30 to -1.31 \\
Glaucoma & +1.49 to -29.79 & -3.43 & -1.21 to -7.64 \\
\hline
\end{tabular}

and NRV $\left(R^{2}=0.79, P<0.01\right)$. Specifically, the model includes both a maximum $y$ measure $(\mathrm{a}+\mathrm{b})$ of normal RNFL thickness and a $y$ intercept (a) representing the RNFL thickness in advanced glaucoma, which incorporates the differences in the nonneuronal components for the RNFL and ONH NRR measures.

For human subjects, the relationship between NRR and RNFL measures were investigated with and without glaucoma suspects. The analysis without glaucoma suspects was performed, as this group consisted of both ocular hypertensive $(n=53)$ individuals and individuals with suspicious optic nerves $(n=21)$ based on NRR appearance, but no detectable RNFL loss noted with ophthalmoscopy, and hence could be a confounder. For both NRV and sMRW measures, an exponential rise to maximum model provided a good description of the data. All parameters for the best fits (Fig. 6) were within the 95\% confidence interval, indicating that the inclusion of glaucoma suspects does not significantly impact the relationship between NRR and RNFL thickness measures. In addition, to eliminate the floor effect of either NRR or RNFL, the relationship between measures was also investigated after removing the bottom one standard deviation of measures. In these truncated data, AICc analysis supported the use of the exponential fit over a linear equation for both NRV (difference in $\mathrm{AICc}=35.09, P<0.01$ ) and sMRW (difference in AICc $=$ 22.42, $P<0.01)$.

Overall, there was good agreement between the data and fits for the two species studied (Fig. 6). The only notable difference between the two species is a larger growth constant ( $t$ ) for the experimental glaucoma group (NRV $t=-12.6$, sMRW $t=-0.009$; Figs. 6A, 6C), compared with patients with glaucoma (NRV $t=-7.32$, sMRW $t=-0.007$; Figs. 6B, 6D). In both cases, the model illustrates that there are essentially two separate phases in the relationship between RNFL and ONH NRR losses over the time course of glaucoma from normal (upper-right data) to advanced neuropathy (lower-left data in each plot). In the early phase, starting with normal structures, there are relatively large changes in the ONH NRR tissue, either sMRW or NRV, compared with RNFL thickness, which seems to best represent the relationship over approximately one-half of the dynamic range of ONH NRR measurements. The second phase includes changes in both RNFL and ONH NRR tissue that ends with the residual tissues of advanced neuropathy, which are likely to be primarily composed of nonneuronal tissue.

\section{Sensitivity and Specificity of ONH and RNFL Measures}

A one-way ANOVA with Tukey post hoc analysis was used to test for differences among normal, glaucoma suspect, and glaucoma eyes. For all three morphological measures, RNFL thickness, $F_{2,262}=105.3, P<0.01 ; \mathrm{NRV}, F_{2,262}=94.8, P<$ 0.01 ; and sMRW, $F_{2,262}=96.2, P<0.01$, there were significant differences among each of the three groups $(P<0.01)$. Figure 
TABLE 4. Mean, SD, and Coefficient of Variation for Axial Length and OCT-Derived Morphological Measures for Nonglaucomatous, Glaucoma Suspect, and Glaucoma Human Eyes

\begin{tabular}{lccc}
\hline & \multicolumn{3}{c}{ Mean (SD), CV \% } \\
\cline { 2 - 4 } & Healthy & Glaucoma Suspect & Glaucoma \\
\hline Axial length, mm & $24.03(1.13), 4.7$ & $24.17(1.07), 4.4$ & $24.29(1.13), 4.7$ \\
Scaled RNFL thickness, $\mu \mathrm{m}$ & $109.9(8.97), 8.1$ & $104.6(11.72), 11.2$ & $84.7(15.67), 18.5$ \\
Scaled RNFL thickness major retinal vessels removed, $\mu \mathrm{m}$ & $94.5(8.37), 8.9$ & $88.6(11.20), 12.6$ & $70.5(14.58), 20.7$ \\
Vascular contribution, $\mu \mathrm{m}$ & $15.3(1.82), 11.9$ & $15.9(2.43), 15.3$ & $14.2(2.45), 17.2$ \\
Scaled minimum rim width, $\mu \mathrm{m}$ & $282.2(44.13), 15.6$ & $225.4(47.41), 21.0$ & $180.8(56.77), 31.4$ \\
Neural rim volume, $\mathrm{mm}^{3}$ & $0.362(0.095), 26.1$ & $0.246(0.087), 35.3$ & $0.179(0.092), 51.1$ \\
\hline
\end{tabular}

7 illustrates the distribution of measures for each of the three measures and groups.

To assess sensitivity and specificity of ONH and RNFL measures, receiver operating characteristic (ROC) curves were generated for global measures from glaucoma and glaucoma suspects to assess the utility of morphological measures. For glaucoma diagnosis (Fig. 7D), the area under the curve (AUC) was not significantly different for RNFL thickness, sMRW, and NRV measures $(P>0.5$, AUC $=0.92,0.92,0.91$, respectively). However, at 95\% sensitivity, RNFL thickness, sMRW, and NRV had a specificity of $76 \%, 65 \%$, and $65 \%$, respectively, indicating that RNFL measures a larger number of true negatives than NRR parameters (dashed line, Fig. 7D). For glaucoma suspects (Fig. 7E), although RNFL thickness measures were useful (AUC $=0.65,95 \% \mathrm{CI} 0.57-0.74, P<0.01)$, sMRW and NRV performed significantly better $(P<0.01$, AUC $=0.81$ and 0.83 , respectively), but were not significant different from each other $(P=0.33)$. However, it is important to note that at $95 \%$ sensitivity, NRR parameters had a specificity of only $38 \%$.

\section{Discussion}

The ONH NRR tissue and RNFL both contain axons of RGCs, and, hence, should show good correspondence across glaucoma severity. The present investigations demonstrate the nonlinear relationship between both NRV and SMRW and RNFL thickness derived from scaled OCT images. In addition, it is especially important for clinical research that the results were remarkably similar for the two study populations (i.e., repeated measures over the time course of experimental glaucoma in nonhuman primates and cross-sectional measures in humans covering the range from normal to moderate glaucoma). To make these structural comparisons, it was important to consider both optical and anatomical factors.
The model of experimental glaucoma in rhesus monkeys presented a rigorous test for the aims of the study because, although the visual systems of human and nonhuman primates are anatomically and functionally similar, there are substantial differences in ocular biometry that need to be taken into consideration for translational studies. Specifically, the rhesus monkey has (1) a shorter eye with higher equivalent power, ${ }^{42,68}$ (2) the optic nerves are smaller, yet may contain a larger number of axons, ${ }^{69,70}$ and (3) the optic nerve has a prominent elliptical shape. ${ }^{46,49}$ For these reasons, it was necessary to develop algorithms for both RNFL and ONH analyses that include compensation for individual differences in ocular biometry and morphology.

For instance, although the standard circumpapillary circular scan provides important information on the relative RNFL thicknesses of the lasered and control eyes in the nonhuman primate ${ }^{31}$ it is important to recognize that the scan path is significantly farther from the rim margin on the nasal and temporal sectors due to the elliptical disc shape, where the RNFL thickness is also thinner. In a previous study, the use of an interpolated elliptical scan path $550 \mu \mathrm{m}$ from, and matching the shape of the BMO, was shown to best correspond with the standard circular clinical scan, and accurately quantify the RNFL in this model. ${ }^{46}$ For human RNFL analysis, the standard clinical 12-degree circular scan and resulting "TSNIT" thickness profile, is the current gold standard. ${ }^{71}$ However, similar to nonhuman primates, RNFL thickness measures are dependent on the physical location of the scan path centered on the optic nerve. ${ }^{72-75}$ Hence, in longer eyes, where the scan path is farther from the ONH, RNFL thickness measures are also less. ${ }^{54,56}$ With the assumption that that the cross-sectional RNFL area or axonal content in the circumpapillary region is constant, ${ }^{59}$ the RNFL thickness can be rescaled to a nominal scan circumference of an emmetropic eye $(10.9 \mathrm{~mm}) .^{24,55,58} \mathrm{In}$ addition, when OCT B-scans of the RNFL are rescaled, most major retinal vessels take on a circular shape. The segmenta-
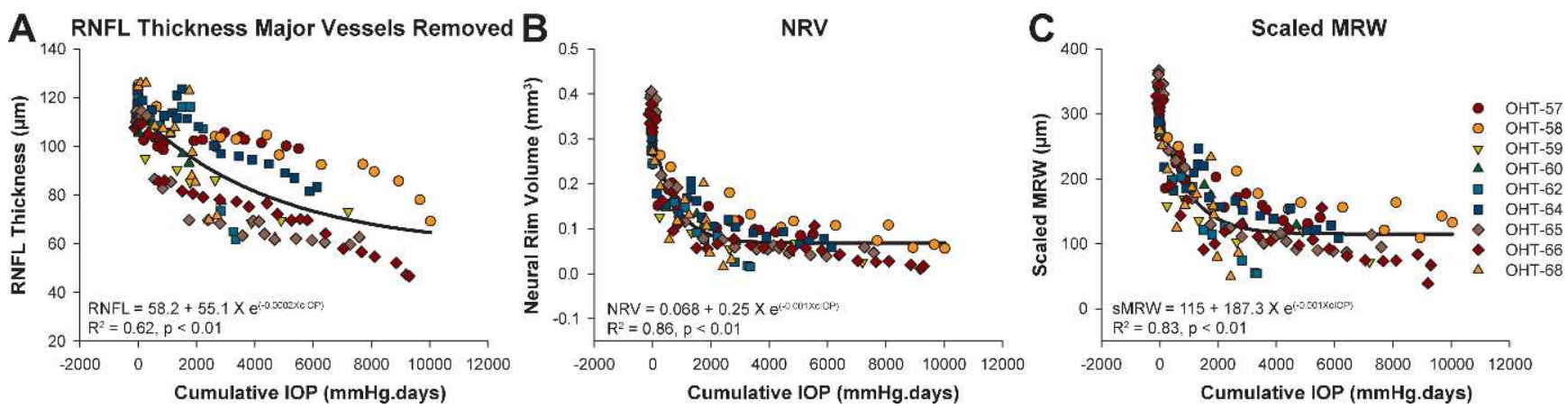

Figure 2. Retinal nerve fiber layer thickness (A), NRV (B), and SMRW (C) as a function of cumulative IOP insult in the nonhuman primate glaucoma model. Each filled symbol represents data from the experimental glaucoma eye of one animal. An exponential decay function was used to describe the rate of change of each metric. 


\section{Detection of Disease and Disease Progression}
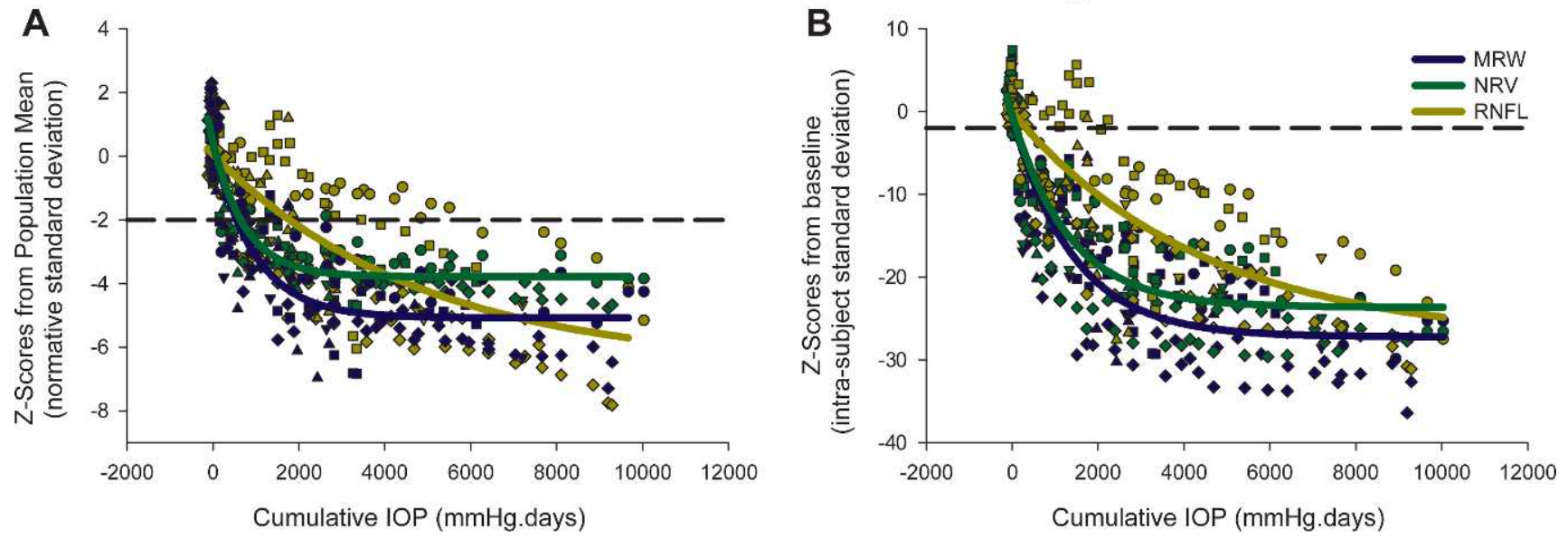

FiguRE 3. (A) Changes in morphological measures of RNFL, NRV, and sMRW expressed as $z$-scores using the population SD as a function of IOP insult. Each morphological measure from the experimental glaucoma eyes is illustrated in a different color. Both ONH parameters reach and exceed 2 SDs (dashed line) before RNFL thickness. (B) Changes in morphological measures expressed as $z$-scores using within-subject SD (Sw) illustrate progression as a function of IOP insult. Similar to (A), both ONH parameters exceed $2.77 \times$ Sw before RNFL measures.

\section{Relationship Between Minimum Rim Width and Neural Rim Volume}
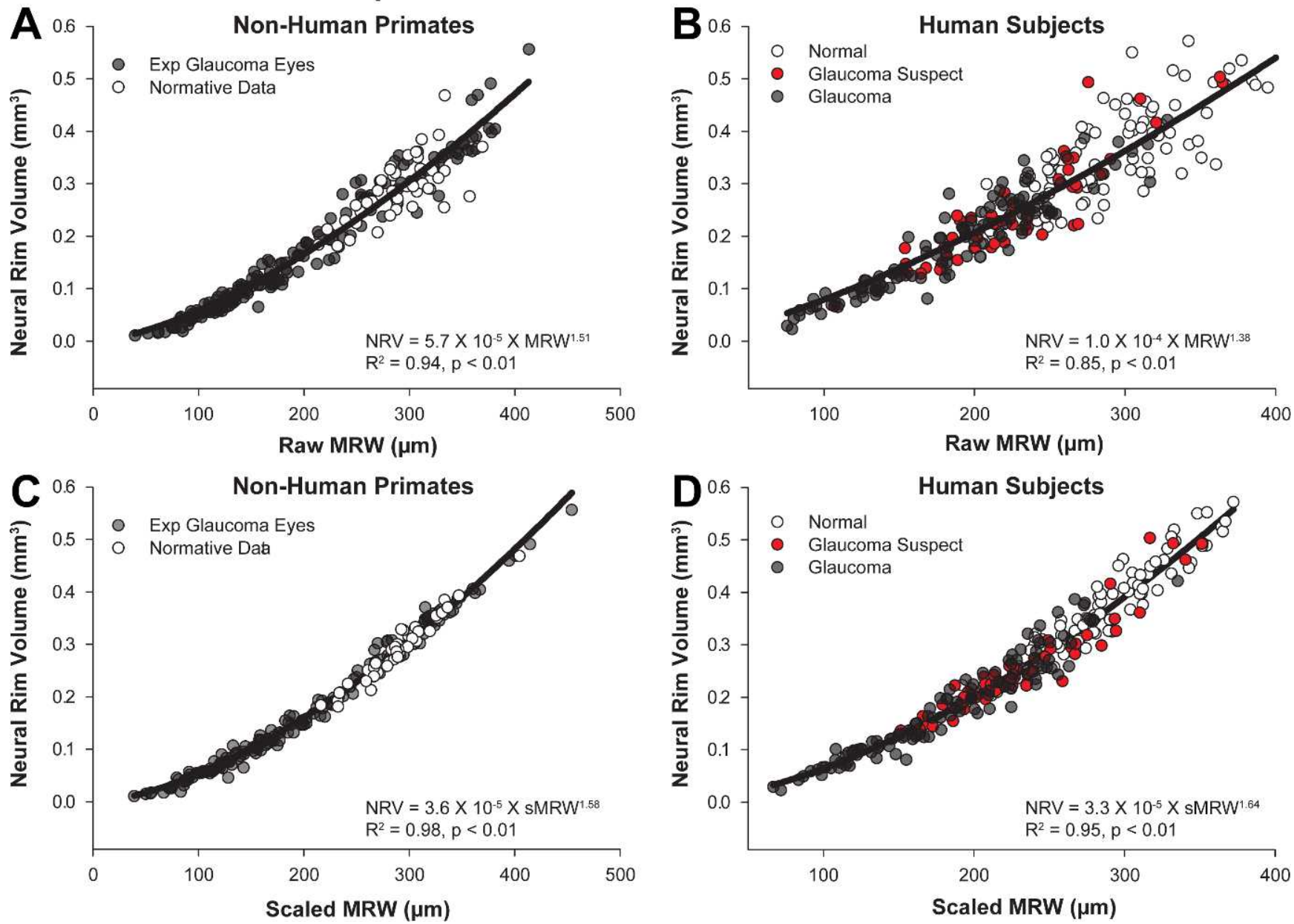

FiguRE 4. The relationship between scaled MRW and NRV in nonhuman primates (A) and human subjects (B). The open circles in (A) represent cross-sectional data from animals used for normative data, whereas the closed circles are all measures from the experimental glaucoma eyes. The data in (B) are cross-sectional data from healthy (open), glaucoma suspects (red), and glaucoma (black) subjects. Plots (C, D) illustrate the improved relationship between the two measures when MRW is scaled to a fixed BMO size. 
Vascular Contribution to Global RNFL Thickness
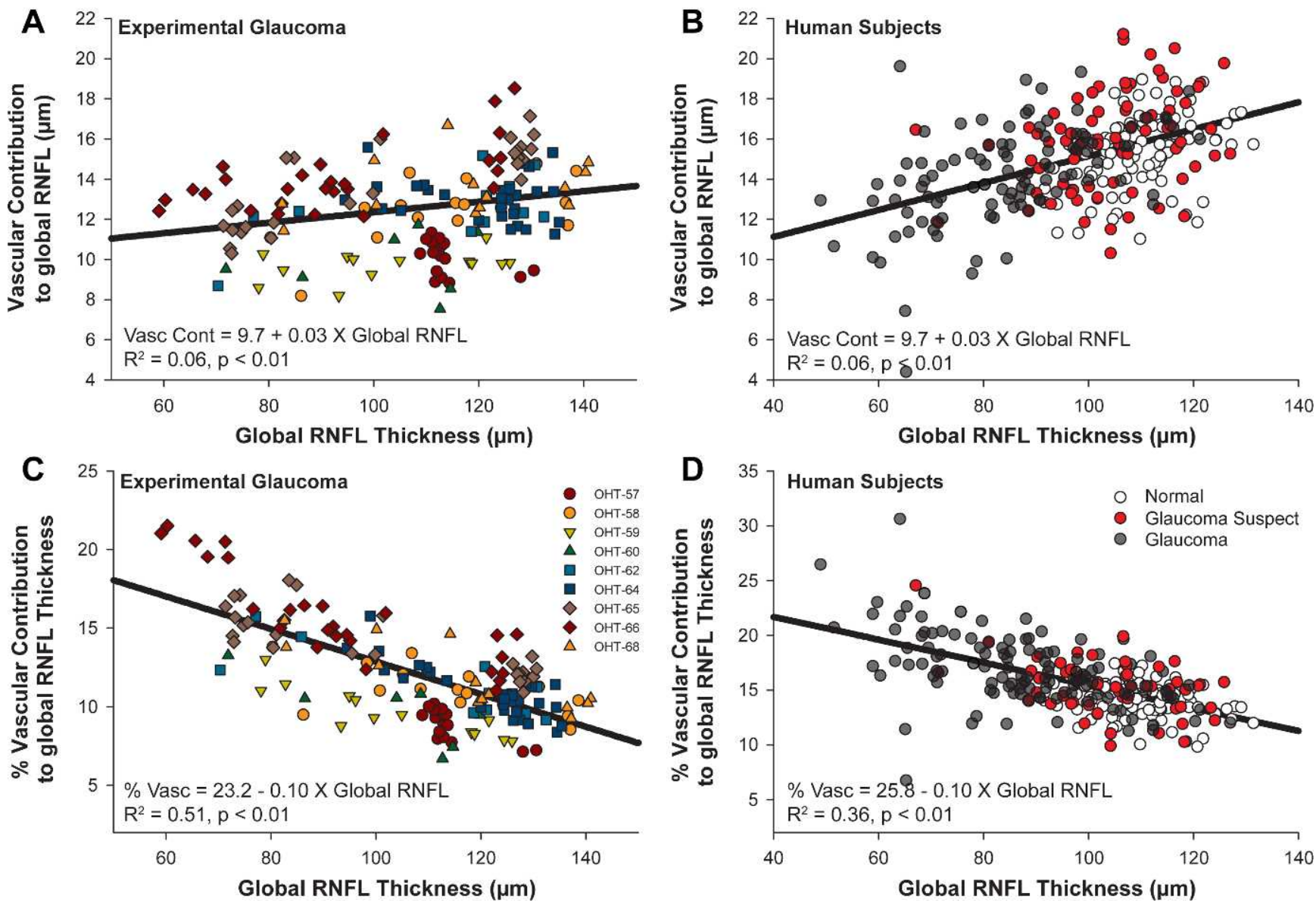

Figure 5. Vascular contribution to the RNFL, when expressed as a thickness, decreases with RNFL in both experimental glaucoma eyes (A), and cross-sectional human subjects data (B). However, the percentage vascular contribution to the RNFL increases with decrease in RNFL thickness (C, D). For nonhuman subjects, each filled symbol represents data from an experimental glaucoma eye (A, C), whereas open, red, and black symbols represent healthy, glaucoma suspect, and glaucoma human subjects $(\mathbf{B}, \mathbf{D})$.

tion protocol used for both human and nonhuman data included all major retinal vasculature that was then subtracted from the RNFL thickness. Although the resulting RNFL thickness has a larger proportion of neural tissue, it still has significant glial and microvascular tissue that cannot be resolved by current OCT technology. ${ }^{22,76,77}$

The results of the present study illustrate the importance of accounting for major retinal vasculature when assessing the RNFL. The major retinal vascular contribution was $9.2 \%$ for rhesus monkeys and $14.0 \%$ in normal human eyes, and similar to those previously reported. ${ }^{23,46}$ It is important to note that the repeatability of vascular contribution was less than that of the RNFL thickness in the nonhuman primate (Table 2), and hence did not have an influence on detecting change over time (data not shown). In both groups, the percent major retinal vascular contribution increased up to $20 \%$ with loss of RNFL. However, the overall thickness contribution of the retinal vasculature decreased with disease progression. The thinning of the vascular contribution was more pronounced in human subjects, most likely reflecting the differences in time courses of experimental and clinical glaucoma. Overall, the changes in the thickness contribution correspond well with previous reports of up to $15 \%^{23,78-80}$ reduction in vessel diameter in glaucoma patients. One factor that was not taken into consideration in the present study, was the influence of age and blood pressure on vascular caliber. ${ }^{81}$ Most studies investigating $\mathrm{ONH}$ morphology in normal and glaucomatous eyes have used confocal scanning laser ophthalmoscopy (CSLO) technology. Overall, measures from CLSO are repeatable $\mathrm{e}^{82-84}$ and provide valuable information for glaucoma diagnosis and management. ${ }^{85-87}$ However, a significant drawback with CLSO is the stability of the reference plane, resulting in higher test-retest variability. ${ }^{88}$ The major step forward with OCT technology has been the identification and use of the relatively stable reference of BMO. ${ }^{48,49,89,90}$ Using this reference, the neural components are usually quantified as either an $\mathrm{RA}^{91}$ or an MRW, ${ }^{50}$ both of which have been shown to have good sensitivity and specificity for glaucoma diagnosis. ${ }^{19,20}$ In theory, an estimate of the RA can be derived from the MRW when the size of the $\mathrm{BMO}$ is known $\left(\mathrm{RA} \approx \pi\left[\mathrm{BMO}\right.\right.$ radius $^{2}-$ (BMO radius $\left.-\mathrm{MRW}^{2}\right]$ ), and the NRV, used in the present study, can be expressed as the RA multiplied by the average thickness within the BMO. As RA is shown to be independent of ethnicity or disc size, ${ }^{91,92}$ the MRW should decrease with increase in disc area, as was illustrated in this and a previous study. ${ }^{19}$ In addition, there should not be a relationship between NRV and disc area, as this measure includes the BMO size in its calculation. Because the outer retina ends at the BMO, the MRW is equivalent to the RNFL thickness at the ONH. Subsequently, the same principles as for scaling RNFL measures can be used to scale the MRW to an average disc size (sMRW), and the resulting scaled measures improved the relationship 

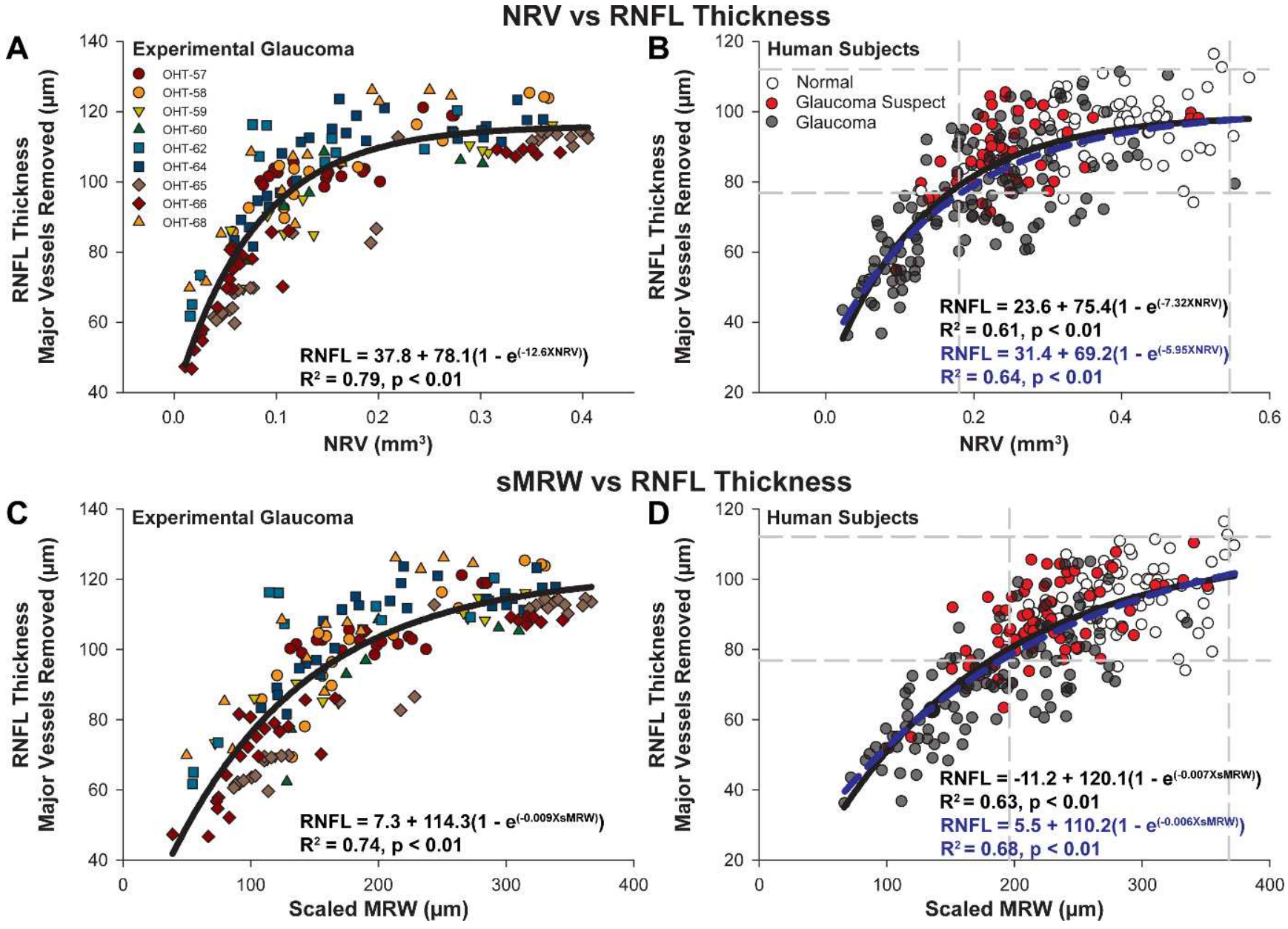

FigURE 6. Both ONH NRR rim parameters decrease before changes in RNFL thickness for all experimental glaucoma eyes (A, C) and the relationship between these measures was best described using an experimental rise to maximum fit. Overall, the trends in the experimental glaucoma group (A, C) were similar to those of the cross-sectional human data (B, D). The gray lines illustrate the $95 \%$ limits for the healthy human subjects. For nonhuman subjects, each filled symbol represents data from an experimental glaucoma eye (A, C), whereas open, red, and black symbols represent healthy, glaucoma suspect, and glaucoma human subjects $(\mathbf{B}, \mathbf{D})$. The dashed blue fit and equations in $(\mathbf{B}, \mathbf{D})$ are after removing glaucoma suspects from the dataset.

with NRV (Fig. 4). This scaling of the MRW to an average disc size was important, as ethnic differences in ONH size were not considered in this study.

Overall, none of the scaled measures for ONH NRR or RNFL were related to axial length in either the human or nonhuman primate groups. However, there are species differences in the relationship between BMO size and neural measures. Specifically, whereas there was no relationship between BMO area and neural parameters in the healthy human subjects, there was a significant relationship in the animal model that corresponds well with most histological studies. ${ }^{53,69,93-95}$

For animals in the experimental glaucoma group, the $\mathrm{ONH}$ NRR parameters (sMRW, NRV) displayed the earliest signs of progression, and the decrease in both sMRW and NRV were similar across animals with cumulative IOP (Figs. 2B, 2C). From the perspective of disease detection, although neural rim parameters have larger population variability, eight of the nine animals had neural rim changes before significant RNFL thinning. In addition, the neural rim volume had decreased by up to $60 \%$ from baseline before RNFL thickness measures falling outside the normative data (Fig. 6). It is important to note that changes in RNFL usually lag those of ONH NRR, in this rapidly progressing model, by one to three scan sessions, corresponding to 2 to 6 weeks. Overall, these findings are consistent with observations from other studies using the nonhuman primate glaucoma model, in which longitudinal changes in both $\mathrm{ONH}$ cup and rim parameters have been demonstrated early in disease state. ${ }^{30,32,63}$

In principal, there also should be good correspondence between these scaled neural measures at the ONH and the RNFL. In fact, this relationship using OCT-derived measures has been reported for glaucoma patients. ${ }^{33}$ However, to the best of our knowledge, this relationship has not been described for longitudinal data, and in healthy and/or glaucoma suspect patients. It was anticipated that the relationship between RNFL thickness and NRV would be nonlinear based on differences in measurement unit, but linear with sMRW that is also quantified in microns. However, both relationships in the experimental glaucoma group were nonlinear, and as with the observations with cumulative IOP, would imply that morphological changes of the ONH NRR precede those of RNFL thickness. In addition, the relationship between ONH parameters and RNFL thickness in the clinical population closely represented those in the experimental glaucoma model. Hence, the data would suggest that the earliest detectable clinical change in glaucoma should be at the ONH in glaucoma suspects with normal RNFL thickness. The lag between ONH changes and RNFL loss in the clinical population, where IOP is significantly lower and 

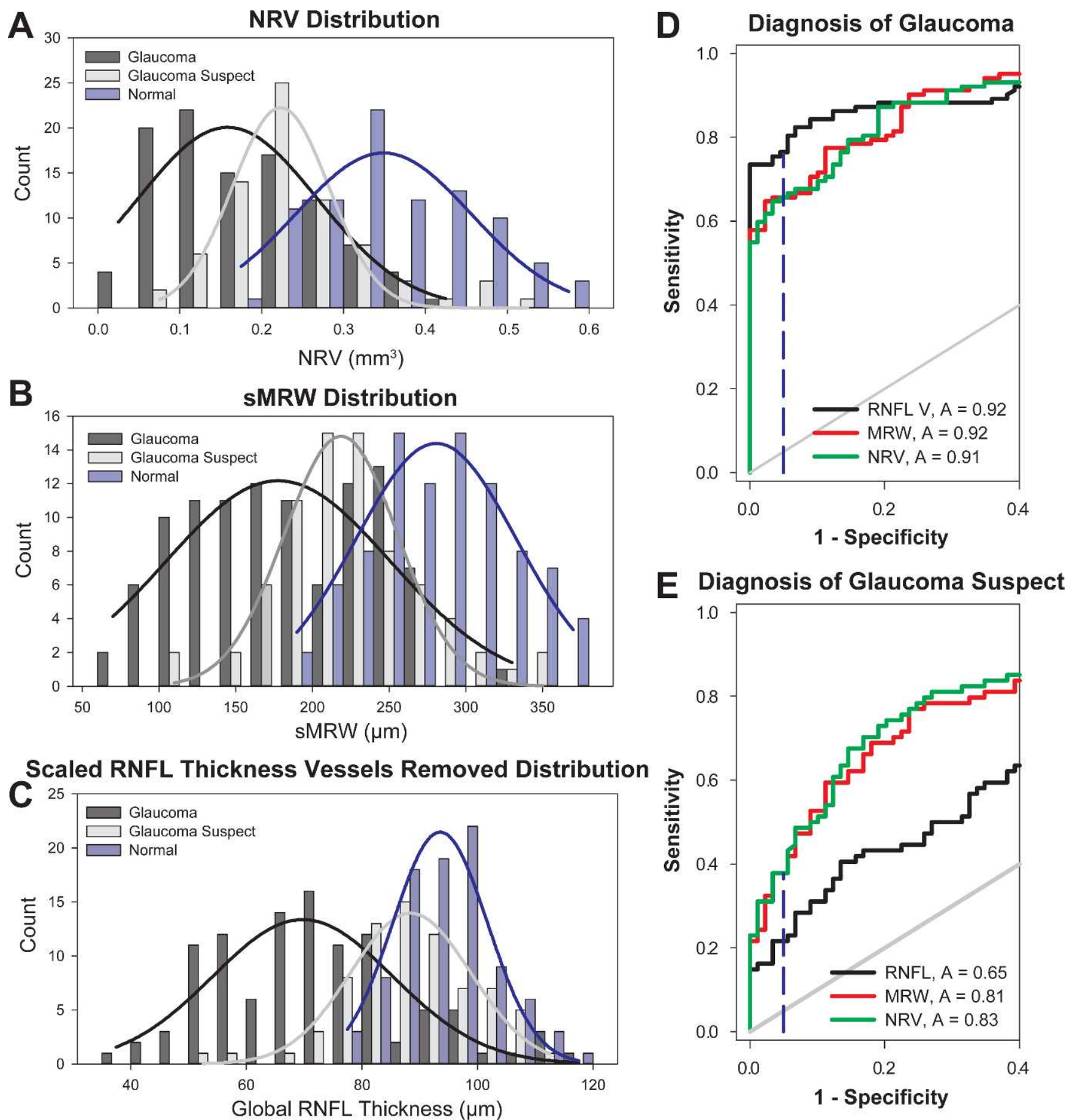

Figure 7. (A-C) Histograms illustrating the distribution and differences for NRV, sMRW, and RNFL thickness measures for healthy, glaucoma suspect, and glaucoma subjects. Sensitivity and specificity assessed using ROC curves for RNFL and ONH parameters comparing healthy with glaucoma (D) and glaucoma suspects (E). Although there is no significant difference for the AUC in (D), at 95\% specificity (dashed blue line), RNFL measures had greater sensitivity. When comparing healthy with glaucoma suspect, ONH NRR measures have a larger AUC in (E); however, at 95\% specificity, the sensitivity is less than $50 \%$.

disease progression is slower, is expected to span over a longer time period, and can be determined only through longitudinal studies.

Although the MRW has been previously shown to have better glaucoma diagnostic value than RNFL, ${ }^{19}$ the AUC for ONH NRR and RNFL measures were not significantly different in the present study. In addition, RNFL thickness measures had higher specificity than ONH NRR parameters at 95\% sensitivity.
It is possible that these differences reflect variances in criteria for glaucoma diagnosis in these different clinics, which also could explain some of the similarities and differences in ROC characteristics in other studies investigating $\mathrm{ONH}$ morphology and RNFL thickness in glaucoma patients. ${ }^{20,96-98}$ The value of ONH parameters in the reported study is best illustrated by the ROCs for the glaucoma suspects. Whereas the AUC for RNFL thickness was only slightly above 0.5 , both sMRW and NRV 
showed significant values. On the other hand, this result may be simply related to the clinical diagnosis of "glaucoma suspect" that is typically based on suspicious ONHs with normal-appearing RNFL, if clinicians are actually judging $\mathrm{ONH}$ NRR tissue to make the diagnosis. Nevertheless, these findings suggest that changes in $\mathrm{ONH}$ neural parameters would be the first indication of conversion of glaucoma suspects to glaucoma patients in the clinical population.

The observed relationship between ONH NRR and RNFL measures could be a result of differences in neural and nonneuronal tissues, or the geometry of the axonal path at the rim margin with disease progression. ${ }^{99-101}$ Specifically, nonneuronal glial and extracellular changes early in the disease are most likely reflected in the loss of ONH NRR tissue, which is subsequently followed by loss of RGC axons. It is possible that neural losses at the ONH precede changes in RNFL thickness, as supported by a recent finding where RNFL thickness loss was manifested only after approximately $10 \%$ of axon loss. ${ }^{102}$ In this instance, it is likely that the Müller glia provide support, and maintain RNFL thickness up to a critical level. The structural support of the Müller cells also may explain why hypodense regions in the RNFL are sometimes seen in the clinical population. ${ }^{103}$

In addition, the nonlinear relationship can be a reflection of the early changes in connective tissue within the peripapillary region. ${ }^{28,104-106}$ Specifically, changes of the sclera, and/or an increase in anterior laminar surface depth, are thought to result in a redistribution of neural tissue. ${ }^{34}$ Hence, it is likely that the axons sampled along the minimum rim width, with the BMO as a reference, have improved laterality and perpendicularity to the measurement distance. In this case, the nonlinear relationship can be viewed in two stages: the first stage, where thickness changes are due to arrangement of axons, and the second stage, where thickness change corresponds to loss of RGC axons.

This study has several limitations. First, the experimental glaucoma group included only animals that were relatively young in age. Hence, the effects of aging on the optic nerve and susceptibility to IOP could not be assessed. Second, the radial scan protocol used to assess the optic nerve morphology comprised only 12 B-scans (15-degree spacing). The number of B-scans was selected based on the practicality of acquiring the data in a clinical setting. However, these scan parameters do not allow for efficient sector-based analysis, and it is also possible that the variability of NRR measures is larger than if a denser scan protocol was used. Third, the data for human subjects, although taken from a observational prospective study, were cross sectional in nature. As additional data are acquired and reported from this and other ongoing prospective studies, it will be important to see if the relationship between ONH NRR and RNFL measures noted in the present study hold. Based on the current study, this will be especially important for assessing change in ocular hypertensive and glaucoma suspect patients.

In conclusion, the findings of this study provide support for the use of multiple OCT-derived morphological measures for the diagnosis and management of primary open angle glaucoma in humans. Although ONH NRR measures may provide valuable information early in the disease, RNFL thickness has the larger dynamic range.

\section{Acknowledgments}

Supported by National Institutes of Health/National Eye Institute Grants EY021761, EY001139, and EY007551, and a John and Rebecca Moores Professorship.

Disclosure: N.B. Patel, None; M. Sullivan-Mee, None; R.S. Harwerth, None

\section{References}

1. Quigley HA. Open-angle glaucoma. N Engl J Med. 1993;328: 1097-1106.

2. The Advanced Glaucoma Intervention Study (AGIS): 7. The relationship between control of intraocular pressure and visual field deterioration. The AGIS Investigators. Am J Ophthalmol. 2000;130:429-440.

3. Gordon MO, Beiser JA, Brandt JD, et al. The Ocular Hypertension Treatment Study: baseline factors that predict the onset of primary open-angle glaucoma. Arch Ophthalmol. 2002;120:714-720.

4. Anderson DR; Normal Tension Glaucoma Study. Collaborative normal tension glaucoma study. Curr Opin Ophthalmol. 2003;14:86-90.

5. Quigley HA, Miller NR, George T. Clinical evaluation of nerve fiber layer atrophy as an indicator of glaucomatous optic nerve damage. Arch Ophthalmol. 1980;98:1564-1571.

6. Sommer A, Miller NR, Pollack I, Maumenee AE, George T. The nerve fiber layer in the diagnosis of glaucoma. Arch Ophthalmol. 1977;95:2149-2156.

7. Weinreb RN, Shakiba S, Sample PA, et al. Association between quantitative nerve fiber layer measurement and visual field loss in glaucoma. Am J Ophthalmol. 1995;120:732-738.

8. Harwerth RS, Carter-Dawson L, Shen F, Smith EL III, Crawford ML. Ganglion cell losses underlying visual field defects from experimental glaucoma. Invest Ophthalmol Vis Sci. 1999;40: 2242-2250.

9. Harwerth RS, Crawford ML, Frishman LJ, Viswanathan S, Smith EL III, Carter-Dawson L. Visual field defects and neural losses from experimental glaucoma. Prog Retin Eye Res. 2002;21:91-125.

10. Keltner JL, Johnson CA, Quigg JM, Cello KE, Kass MA, Gordon MO. Confirmation of visual field abnormalities in the Ocular Hypertension Treatment Study. Ocular Hypertension Treatment Study Group. Arch Ophthalmol. 2000;118:11871194.

11. Reus NJ, Lemij HG, Garway-Heath DF, et al. Clinical assessment of stereoscopic optic disc photographs for glaucoma: the European Optic Disc Assessment Trial. Ophthalmology. 2010;117:717-723.

12. Teitelbaum BA, Haefs R, Connor D. Interobserver variability in the estimation of the cup/disk ratio among observers of differing educational background. Optometry. 2001;72:729732.

13. Schuman JS, Hee MR, Puliafito CA, et al. Quantification of nerve fiber layer thickness in normal and glaucomatous eyes using optical coherence tomography. Arch Ophthalmol. 1995;113:586-596.

14. Schuman JS, Pedut-Kloizman T, Hertzmark E, et al. Reproducibility of nerve fiber layer thickness measurements using optical coherence tomography. Ophthalmology. 1996;103: 1889-1898.

15. Blumenthal EZ, Williams JM, Weinreb RN, Girkin CA, Berry CC, Zangwill LM. Reproducibility of nerve fiber layer thickness measurements by use of optical coherence tomography. Ophthalmology. 2000;107:2278-2282.

16. Carpineto P, Nubile M, Agnifili L, et al. Reproducibility and repeatability of Cirrus HD-OCT peripapillary retinal nerve fibre layer thickness measurements in young normal subjects. Ophthalmologica. 2012;227:139-145.

17. Budenz DL, Fredette MJ, Feuer WJ, Anderson DR. Reproducibility of peripapillary retinal nerve fiber thickness measurements with stratus OCT in glaucomatous eyes. Ophthalmology. 2008;115:661-666.

18. Mwanza JC, Chang RT, Budenz DL, et al. Reproducibility of peripapillary retinal nerve fiber layer thickness and optic nerve head parameters measured with cirrus HD-OCT in 
glaucomatous eyes. Invest Ophthalmol Vis Sci. 2010;51: 5724-5730.

19. Chauhan BC, O'Leary N, Almobarak FA, et al. Enhanced detection of open-angle glaucoma with an anatomically accurate optical coherence tomography-derived neuroretinal rim parameter. Ophthalmology. 2013;120:535-543.

20. Mwanza JC, Oakley JD, Budenz DL, Anderson DR; Cirrus Optical Coherence Tomography Normative Database Study Group. Ability of cirrus HD-OCT optic nerve head parameters to discriminate normal from glaucomatous eyes. Ophthalmology. 2011;118:241-248.

21. Uchida H, Brigatti L, Caprioli J. Detection of structural damage from glaucoma with confocal laser image analysis. Invest Ophthalmol Vis Sci. 1996;37:2393-2401.

22. Ogden TE. Nerve fiber layer of the primate retina: thickness and glial content. Vision Res. 1983;23:581-587.

23. Hood DC, Fortune B, Arthur SN, et al. Blood vessel contributions to retinal nerve fiber layer thickness profiles measured with optical coherence tomography. J Glaucoma. 2008; 17:519-528.

24. Patel NB, Wheat JL, Rodriguez A, Tran V, Harwerth RS. Agreement between retinal nerve fiber layer measures from Spectralis and Cirrus spectral domain OCT. Optom Vis Sci. 2012;89:E652-E666.

25. Harwerth RS, Crawford ML. The relationship between perimetric and metabolic defects caused by experimental glaucoma. In: Henson $\mathrm{DB}$, Wall $\mathrm{M}$, eds. Perimetric Update 2002/2003. The Hague: Kugler Publication; 2002:175-186.

26. Harwerth RS. Primate glaucoma models. In: Pang IH, Clark AF, eds. Animal Models for Retinal Disease. New York, NY: Humana Press; 2010:165-189.

27. Harwerth RS, Smith EL III, DeSantis L. Behavioral perimetry in monkeys. Invest Ophthalmol Vis Sci. 1993;34:31-40.

28. Burgoyne CF, Downs JC, Bellezza AJ, Suh JK, Hart RT. The optic nerve head as a biomechanical structure: a new paradigm for understanding the role of IOP-related stress and strain in the pathophysiology of glaucomatous optic nerve head damage. Prog Retin Eye Res. 2005;24:39-73.

29. Burgoyne CF, Downs JC, Bellezza AJ, Hart RT. Threedimensional reconstruction of normal and early glaucoma monkey optic nerve head connective tissues. Invest Ophthalmol Vis Sci. 2004;45:4388-4399.

30. Strouthidis NG, Fortune B, Yang H, Sigal IA, Burgoyne CF. Longitudinal change detected by spectral domain optical coherence tomography in the optic nerve head and peripapillary retina in experimental glaucoma. Invest $O p b$ thalmol Vis Sci. 2011;52:1206-1219.

31. Harwerth RS, Vilupuru AS, Rangaswamy NV, Smith EL III. The relationship between nerve fiber layer and perimetry measurements. Invest Ophthalmol Vis Sci. 2007;48:763-773.

32. He L, Yang H, Gardiner SK, et al. Longitudinal detection of optic nerve head changes by spectral domain optical coherence tomography in early experimental glaucoma. Invest Ophthalmol Vis Sci. 2014;55:574-586.

33. Gardiner SK, Ren R, Yang H, Fortune B, Burgoyne CF, Demirel SA. Method to estimate the amount of neuroretinal rim tissue in glaucoma: comparison with current methods for measuring rim area. Am J Ophthalmol. 2014;157:540-549.

34. Strouthidis NG, Fortune B, Yang H, Sigal IA, Burgoyne CF. Effect of acute intraocular pressure elevation on the monkey optic nerve head as detected by spectral domain optical coherence tomography. Invest Ophthalmol Vis Sci. 2011;52: 9431-9437.

35. Burke JA, Potter DE. The ocular effects of xylazine in rabbits, cats, and monkeys. J Ocul Pharmacol. 1986;2:9-21.

36. Frishman LJ, Shen FF, Du L, et al. The scotopic electroretinogram of macaque after retinal ganglion cell loss from experimental glaucoma. Invest Ophthalmol Vis Sci. 1996;37: 125-141.

37. Quigley HA, Hohman RM. Laser energy levels for trabecular meshwork damage in the primate eye. Invest Ophthalmol Vis Sci. 1983;24:1305-1307.

38. Harwerth RS, Smith EL III, DeSantis L. Experimental glaucoma: perimetric field defects and intraocular pressure. J Glaucoma. 1997;6:390-401.

39. Gaasterland D, Kupfer C. Experimental glaucoma in the rhesus monkey. Invest Ophthalmol. 1974;13:455-457.

40. Rasmussen CA, Kaufman PL. Primate glaucoma models. $J$ Glaucoma. 2005;14:311-314.

41. Yang H, Thompson H, Roberts MD, Sigal IA, Downs JC, Burgoyne CF. Deformation of the early glaucomatous monkey optic nerve head connective tissue after acute IOP elevation in 3-D histomorphometric reconstructions. Invest Ophthalmol Vis Sci. 2011;52:345-363.

42. Qiao-Grider Y, Hung LF, Kee CS, Ramamirtham R, Smith EL III. Normal ocular development in young rhesus monkeys (Macaca mulatta). Vision Res. 2007;47:1424-1444.

43. Atchison DA, Markwell EL, Kasthurirangan S, Pope JM, Smith G, Swann PG. Age-related changes in optical and biometric characteristics of emmetropic eyes. J Vis. 2008;8(29):21-20.

44. Atchison DA. Age-related paraxial schematic emmetropic eyes. Ophthalmic Physiol Opt. 2009;29:58-64.

45. Bennett AG, Rabbetts RB. The schematic eye. In: Bennett AG, Rabbetts RB, eds. Clinical Visual Optics. London: Butterworths; 1989:249-274.

46. Patel NB, Luo X, Wheat JL, Harwerth RS. Retinal nerve fiber layer assessment: area versus thickness measurements from elliptical scans centered on the optic nerve. Invest Ophthalmol Vis Sci. 2011;52:2477-2489.

47. Patel NB, Garcia B, Harwerth RS. Influence of anterior segment power on the scan path and RNFL thickness using SD-OCT. Invest Ophthalmol Vis Sci. 2012;53:5788-5798.

48. Strouthidis NG, Yang H, Reynaud JF, et al. Comparison of clinical and spectral domain optical coherence tomography optic disc margin anatomy. Invest Ophthalmol Vis Sci. 2009; 50:4709-4718.

49. Strouthidis NG, Yang H, Fortune B, Downs JC, Burgoyne CF. Detection of optic nerve head neural canal opening within histomorphometric and spectral domain optical coherence tomography data sets. Invest Ophthalmol Vis Sci. 2009;50: 214-223.

50. Reis AS, O'Leary N, Yang $\mathrm{H}$, et al. Influence of clinically invisible, but optical coherence tomography detected, optic disc margin anatomy on neuroretinal rim evaluation. Invest Ophthalmol Vis Sci. 2012;53:1852-1860.

51. Reis AS, Sharpe GP, Yang H, Nicolela MT, Burgoyne CF, Chauhan BC. Optic disc margin anatomy in patients with glaucoma and normal controls with spectral domain optical coherence tomography. Ophthalmology. 2012;119:738-747.

52. Tsutsumi T, Tomidokoro A, Araie M, Iwase A, Sakai H, Sawaguchi S. Planimetrically determined vertical cup/disc and rim width/disc diameter ratios and related factors. Invest Ophthalmol Vis Sci. 2012;53:1332-1340.

53. Jonas JB, Schmidt AM, Muller-Bergh JA, Schlotzer-Schrehardt UM, Naumann GO. Human optic nerve fiber count and optic disc size. Invest Ophthalmol Vis Sci. 1992;33:2012-2018.

54. Budenz DL, Anderson DR, Varma R, et al. Determinants of normal retinal nerve fiber layer thickness measured by Stratus OCT. Ophthalmology. 2007;114:1046-1052.

55. Savini G, Barboni P, Parisi V, Carbonelli M. The influence of axial length on retinal nerve fibre layer thickness and opticdisc size measurements by spectral-domain OCT. $\mathrm{Br} J$ Ophthalmol. 2012;96:57-61. 
56. Varma R, Skaf M, Barron E. Retinal nerve fiber layer thickness in normal human eyes. Ophthalmology. 1996;103:21142119.

57. Curcio CA, Allen KA. Topography of ganglion cells in human retina. J Comp Neurol. 1990;300:5-25.

58. Kang SH, Hong SW, Im SK, Lee SH, Ahn MD. Effect of myopia on the thickness of the retinal nerve fiber layer measured by Cirrus HD optical coherence tomography. Invest Ophthalmol Vis Sci. 2010;51:4075-4083.

59. Bayraktar S, Bayraktar Z, Yilmaz OF. Influence of scan radius correction for ocular magnification and relationship between scan radius with retinal nerve fiber layer thickness measured by optical coherence tomography. J Glaucoma. 2001;10: 163-169.

60. Burnham KP, Anderson DR. Multimodel inference-understanding AIC and BIC in model selection. Sociol Method Res. 2004;33:261-304.

61. Bland JM, Altman DG. Measurement error. BMJ. 1996;313: 744.

62. Li H, Healey PR, Tariq YM, Teber E, Mitchell P. Symmetry of optic nerve head parameters measured by the Heidelberg retina tomograph 3 in healthy eyes: the Blue Mountains Eye study. Am J Ophthalmol. 2013;155:518-523.e1.

63. Fortune B, Reynaud J, Wang L, Burgoyne CF. Does optic nerve head surface topography change prior to loss of retinal nerve fiber layer thickness: a test of the site of injury hypothesis in experimental glaucoma. PLoS One. 2013;8:e77831.

64. Quigley HA, Addicks EM, Green WR, Maumenee AE. Optic nerve damage in human glaucoma. II. The site of injury and susceptibility to damage. Arch Ophthalmol. 1981;99:635649.

65. Nickells RW. From ocular hypertension to ganglion cell death: a theoretical sequence of events leading to glaucoma. Can J Ophthalmol. 2007;42:278-287.

66. Nickells RW, Howell GR, Soto I, John SW. Under pressure: cellular and molecular responses during glaucoma, a common neurodegeneration with axonopathy. Ann Rev Neurosci. 2012;35:153-179.

67. Hernandez MR. The optic nerve head in glaucoma: role of astrocytes in tissue remodeling. Prog Retin Eye Res. 2000;19: 297-321.

68. Lapuerta P, Schein SJ. A four-surface schematic eye of macaque monkey obtained by an optical method. Vision Res. 1995;35:2245-2254.

69. Quigley HA, Coleman AL, Dorman-Pease ME. Larger optic nerve heads have more nerve fibers in normal monkey eyes. Arch Ophthalmol. 1991;109:1441-1443.

70. Cull G, Cioffi GA, Dong J, Homer L, Wang L. Estimating normal optic nerve axon numbers in non-human primate eyes. J Glaucoma. 2003;12:301-306.

71. Bussel II, Wollstein G, Schuman JS. OCT for glaucoma diagnosis, screening and detection of glaucoma progression. Br J Ophthalmol. 2014;98(suppl 2):ii15-19.

72. Savini G, Barboni P, Carbonelli M, Zanini M. The effect of scan diameter on retinal nerve fiber layer thickness measurement using stratus optic coherence tomography. Arch Ophthalmol. 2007;125:901-905.

73. Vizzeri G, Bowd C, Medeiros FA, Weinreb RN, Zangwill LM. Effect of improper scan alignment on retinal nerve fiber layer thickness measurements using Stratus optical coherence tomograph. J Glaucoma. 2008;17:341-349.

74. Vizzeri G, Bowd C, Medeiros FA, Weinreb RN, Zangwill LM. Effect of signal strength and improper alignment on the variability of stratus optical coherence tomography retinal nerve fiber layer thickness measurements. Am J Ophthalmol. 2009;148:249-255.e1.
75. Gabriele ML, Ishikawa H, Wollstein G, et al. Optical coherence tomography scan circle location and mean retinal nerve fiber layer measurement variability. Invest Ophthalmol Vis Sci. 2008;49:2315-2321.

76. Ogden TE. Nerve fiber layer of the macaque retina: retinotopic organization. Invest Ophthalmol Vis Sci. 1983; 24:85-98.

77. Scoles D, Gray DC, Hunter JJ, et al. In-vivo imaging of retinal nerve fiber layer vasculature: imaging histology comparison. BMC Ophthalmol. 2009;9:1-9.

78. Rader J, Feuer WJ, Anderson DR. Peripapillary vasoconstriction in the glaucomas and the anterior ischemic optic neuropathies. Am J Ophthalmol. 1994;117:72-80.

79. Amerasinghe N, Aung T, Cheung N, et al. Evidence of retinal vascular narrowing in glaucomatous eyes in an Asian population. Invest Ophthalmol Vis Sci. 2008;49:5397-5402.

80. Mitchell P, Leung H, Wang JJ, et al. Retinal vessel diameter and open-angle glaucoma: the Blue Mountains Eye Study. Ophthalmology. 2005;112:245-250.

81. Leung H, Wang JJ, Rochtchina E, et al. Relationships between age, blood pressure, and retinal vessel diameters in an older population. Invest Ophthalmol Vis Sci. 2003;44:2900-2904.

82. Kruse FE, Burk RO, Volcker HE, Zinser G, Harbarth U. Reproducibility of topographic measurements of the optic nerve head with laser tomographic scanning. Ophthalmology. 1989;96:1320-1324.

83. Dreher AW, Tso PC, Weinreb RN. Reproducibility of topographic measurements of the normal and glaucomatous optic nerve head with the laser tomographic scanner. Am J Ophthalmol. 1991;111:221-229.

84. Chauhan BC, LeBlanc RP, McCormick TA, Rogers JB. Testretest variability of topographic measurements with confocal scanning laser tomography in patients with glaucoma and control subjects. Am J Ophthalmol. 1994;118:9-15.

85. Wollstein G, Garway-Heath DF, Hitchings RA. Identification of early glaucoma cases with the scanning laser ophthalmoscope. Ophthalmology. 1998;105:1557-1563.

86. Wollstein G, Garway-Heath DF, Fontana L, Hitchings RA. Identifying early glaucomatous changes. Comparison between expert clinical assessment of optic disc photographs and confocal scanning ophthalmoscopy. Ophthalmology. 2000;107:2272-2277.

87. Miglior S, Casula M, Guareschi M, Marchetti I, Iester M, Orzalesi N. Clinical ability of Heidelberg retinal tomograph examination to detect glaucomatous visual field changes. Ophthalmology. 2001;108:1621-1627.

88. Tan JC, Garway-Heath DF, Fitzke FW, Hitchings RA. Reasons for rim area variability in scanning laser tomography. Invest Ophthalmol Vis Sci. 2003;44:1126-1131.

89. Downs JC, Yang H, Girkin C, et al. Three-dimensional histomorphometry of the normal and early glaucomatous monkey optic nerve head: neural canal and subarachnoid space architecture. Invest Ophthalmol Vis Sci. 2007;48: 3195-3208.

90. Strouthidis NG, Yang H, Downs JC, Burgoyne CF. Comparison of clinical and three-dimensional histomorphometric optic disc margin anatomy. Invest Ophthalmol Vis Sci. 2009;50: 2165-2174.

91. Knight OJ, Girkin CA, Budenz DL, Durbin MK, Feuer WJ; Cirrus OCT Normative Database Study Group. Effect of race, age, and axial length on optic nerve head parameters and retinal nerve fiber layer thickness measured by Cirrus HDOCT. Arch Ophthalmol. 2012;130:312-318.

92. Girkin CA, McGwin G Jr, Long C, DeLeon-Ortega J, Graf CM, Everett AW. Subjective and objective optic nerve assessment in African Americans and whites. Invest Ophthalmol Vis Sci. 2004; $45: 2272-2278$. 
93. Mikelberg FS, Yidegiligne HM, White VA, Schulzer M. Relation between optic nerve axon number and axon diameter to scleral canal area. Ophthalmology. 1991;98:6063.

94. Jonas JB, Muller-Bergh JA, Schlotzer-Schrehardt UM, Naumann GO. Histomorphometry of the human optic nerve. Invest Ophthalmol Vis Sci. 1990;31:736-744.

95. Balazsi AG, Rootman J, Drance SM, Schulzer M, Douglas GR. The effect of age on the nerve fiber population of the human optic nerve. Am J Ophthalmol. 1984;97:760-766.

96. Li S, Wang X, Li S, Wu G, Wang N. Evaluation of optic nerve head and retinal nerve fiber layer in early and advance glaucoma using frequency-domain optical coherence tomography. Graefes Arch Clin Exp Ophthalmol. 2010;248:429434.

97. Sung KR, Na JH, Lee Y. Glaucoma diagnostic capabilities of optic nerve head parameters as determined by Cirrus HD optical coherence tomography. J Glaucoma. 2012;21:498504.

98. Lisboa R, Leite MT, Zangwill LM, Tafreshi A, Weinreb RN, Medeiros FA. Diagnosing preperimetric glaucoma with spectral domain optical coherence tomography. Ophthalmology. 2012;119:2261-2269.

99. Varela HJ, Hernandez MR. Astrocyte responses in human optic nerve head with primary open-angle glaucoma. $J$ Glaucoma. 1997;6:303-313.
100. Hernandez MR, Miao H, Lukas T. Astrocytes in glaucomatous optic neuropathy. Prog Brain Res. 2008;173:353-373.

101. Tezel G; Fourth ARVO/Pfizer Ophthalmics Research Institute Conference Working Group. The role of glia, mitochondria, and the immune system in glaucoma. Invest Ophthalmol Vis Sci. 2009;50:1001-1012.

102. Cull GA, Reynaud J, Wang L, Cioffi GA, Burgoyne CF, Fortune B. Relationship between orbital optic nerve axon counts and retinal nerve fiber layer thickness measured by spectral domain optical coherence tomography. Invest Ophthalmol Vis Sci. 2012;53:7766-7773.

103. Xin D, Talamini CL, Raza AS, et al. Hypodense regions (holes) in the retinal nerve fiber layer in frequency-domain OCT scans of glaucoma patients and suspects. Invest Ophthalmol Vis Sci. 2011;52:7180-7186.

104. Girard MJ, Downs JC, Bottlang M, Burgoyne CF, Suh JK. Peripapillary and posterior scleral mechanics-part II: experimental and inverse finite element characterization. $J$ Biomech Eng. 2009;131:051012.

105. Yan DB, Coloma FM, Metheetrairut A, Trope GE, Heathcote JG, Ethier CR. Deformation of the lamina cribrosa by elevated intraocular pressure. Br J Ophthalmol. 1994;78:643-648.

106. Bellezza AJ, Rintalan CJ, Thompson HW, Downs JC, Hart RT, Burgoyne CF. Deformation of the lamina cribrosa and anterior scleral canal wall in early experimental glaucoma. Invest Ophthalmol Vis Sci. 2003;44:623-637. 